\title{
CONSOLIDAÇÃO DO FARMACÊUTICO NA PRÁTICA CLÍNICA
}

\section{PHARMACEUTICAL CONSOLIDATION IN CLINICAL PRACTICE}

\section{CAROLINE GRIBNER1}

1 - Doutoranda do Programa de Pós-Graduação em Ciências Farmacêuticas - Universidade Federal do Paraná (UFPR). Curitiba/PR - Brasil.

Autor para correspondência: carol_gribner@yahoo.com.br

\section{RESUMO:}

A participação do farmacêutico na equipe multiprofissional em um ambiente hospitalar vem crescendo expressivamente com o passar dos anos, o que contribui com o fortalecimento e consolidação da atenção farmacêutica e sua prática clínica. O objetivo deste estudo foi demonstrar que o profissional farmacêutico pode realizar o acompanhamento do paciente, contribuindo na farmacoterapia, evitando erros e acidentes associados ao uso incorreto de medicamentos, além de evidenciar o crescimento de artigos que reforçam a profissão na prática clínica. Os dados foram obtidos por meio de uma revisão bibliográfica, tendo como base periódicos publicados nas bases de dados Scielo, Portal da CAPES e PubMed. Diversos estudos demonstram a importância da intervenção e acompanhamento clínico realizado pelo farmacêutico que compõe a equipe multidisciplinar de estabelecimentos de saúde como hospitais, as publicações de artigos que abordam esta temática apresentaram crescimento expressivo nas últimas décadas. Com o decorrer dos anos foi possível verificar os avanços relacionados à regulamentação e organização dos serviços farmacêuticos, bem como a crescente da participação do profissional farmacêutico no cuidado a saúde dos pacientes a nível nacional e mundial.

Palavras-chave: Atenção Farmacêutica. Farmácia Clínica. Farmacêutico.

\section{ABSTRACT:}

The participation of the pharmacist in the multiprofessional team in a hospital environment has been increasing significantly over the years, which contributes to the strengthening and consolidation of pharmaceutical care and its clinical practice. The objective of this study was to demonstrate that the pharmacist is able to follow the patient, contribute to pharmacotherapy, uncover the errors and risk factors related to the incorrect use of medications and the process of conducting research in clinical practice, besides evidencing the growth of articles that reinforce the profession in clinical practice. The data were reached through the bibliographic review, based on the Scielo databases, CAPES Portal, PubMed. Several studies demonstrate the importance of clinical intervention and follow up performed by pharmacists, who make up the multidisciplinary team of health care facilities such as hospitals, the publications of articles dealing with this theme have shown significant growth in the last decades. Over the years it has been possible to verify the advances related to the regulation and organization of pharmaceutical services, as well as the increasing participation of the pharmaceutical professional in the health care of patients at national and worldwide level.

Key words: Pharmaceutical Care. Pharmacy Clinic. Pharmaceutical. 


\section{INTRODUÇÃO}

A participação do profissional farmacêutico em instituições de saúde como hospitais e unidades básicas de saúde vem crescendo expressivamente nos últimos anos, o que contribui com o seu fortalecimento e consolidação na prática clínica. Uma de várias outras funções do farmacêutico neste ambiente é o emprego da farmácia clínica, a qual é utilizada com o intuito de proporcionar o uso racional e seguro dos medicamentos (ANGONESI; SEVALHO, 2010).

Esta prática é regulamentada e respaldada por meio de duas Resoluções publicadas pelo Conselho Federal de Farmácia, a Resolução n 585 de 29 de agosto de 2013 que regulamenta as atribuições clínicas do farmacêutico e a Resolução n`586, de 29 de agosto de 2013, regulamenta a prescrição farmacêutica e dá outras providências relacionadas ao exercício da profissão farmacêutica (BRASIL, 2013a; BRASIL, 2013b). Outra conquista importante foi obtida por meio da Lei $n^{\circ} 13.021$, a qual muda o conceito de farmácia no Brasil. Essa lei possibilitou ao farmacêutico a prestação de cuidados direto com o paciente, por transformar farmácias e drogarias em unidades de prestação de assistência farmacêutica e assistência à saúde (BRASIL, 2014).

A aplicação da farmácia clínica mostra-se como uma prática na qual os farmacêuticos prestam cuidados aos pacientes com o objetivo de otimizar a farmacoterapia empregada. Como recurso pode-se optar por diferentes abordagens que envolvem a revisão da prescrição clínica, entrevistas e consultas, aconselhamentos e acompanhamento aos internados, discursão de casos clínicos com outros profissionais da equipe, entre outros (CARREÑO, et al., 2012; OKUMURA, et al., 2016).

Como resultado de todo este processo, tem-se o desenvolvimento de um plano de atenção farmacêutica, que busca elucidar situações adversas que podem ser presenciadas durante o tratamento do paciente. Esta intervenção farmacêutica, realizada de modo planejado possibilita ao profissional resolver e prevenir problemas que poderiam interferir na farmacoterapia do paciente (COSTA, et al., 2017).

Como ferramenta aliada à prática clínica tem-se a atenção farmacêutica, que possui ações voltadas à promoção, proteção e recuperação da saúde do paciente. De maneira complementar possui como foco melhorar a qualidade de vida do paciente, por meio da busca pela cura e prevenção de doenças, redução de sintomas, entre outros (OPAS/OMS, 2002).

Desta maneira tem-se como objetivo geral demonstrar a importância do farmacêutico clínico no cuidado e na assistência à saúde do paciente. Analisando de forma complementar 
as atribuições do farmacêutico no acompanhamento clínico do paciente e o reflexo causado no crescimento do número de publicações de artigos que abordem as práticas de cuidados farmacêuticos.

Para a realização desta pesquisa optou-se por utilizar metodologia qualitativa, realizada por meio de uma revisão bibliográfica. Foram utilizadas as seguintes bases de dados: Scielo (Scientífic Eletronic Library Online), Portal da CAPES (Coordenação de Aperfeiçoamento de Pessoas de Nível Superior) e PubMed. Após a identificação dos conteúdos relacionados à proposta inicial foi realizada uma avaliação de publicações sobre o tema que envolve os objetivos listados.

\section{METODOLOGIA}

Trata-se de um estudo qualitativo realizado por meio de uma revisão bibliográfica, tendo como base livros e artigos obtidos em sites e periódicos relacionados à área da saúde.

A pesquisa qualitativa possibilita estudar de maneira aprofundada diferentes assuntos (GERHARDT, SILVEIRA, 2009). Por meio do levantamento de dados é possível que o pesquisador consiga informações sobre os questionamentos que se procura responder (FONSECA, 2002).

Foram utilizadas as seguintes bases de dados: Scielo (Scientífic Eletronic Library Online), Portal da CAPES (Coordenação de Aperfeiçoamento de Pessoas de Nível Superior) e PubMed, sendo selecionados os artigos que relacionem o farmacêutico na prática clínica. Os descritores analisados foram: farmácia clínica e intervenção farmacêutica. Sendo delimitado um período inicial onde foram publicados os primeiros artigos até dezembro de 2018, para englobar estudos que estejam na linha da temática proposta.

Após a identificação dos conteúdos relacionados à proposta inicial, foi realizada uma revisão sobre a atenção farmacêutica aliada à prática clínica. Posteriormente foi realizado um levantamento que demonstra a evolução das publicações sobre o tema proposto que envolve os objetivos listados. O levantamento dos artigos publicados foi realizado por meio da base de dados Pubmed tendo como descritores "hospital clinical pharmacy" e "hospital clinical pharmacy" AND "Brazil" a fim de verificar a evolução do número de publicações nessa área a nível mundial e nacional. Foram considerados os primeiros artigos publicados até os mais recentes, publicados no ano de 2018. 


\section{O FARMACÊUTICO NA PRÁTICA CLÍNICA}

\subsection{Farmácia clínica}

A farmácia clínica teve seu início nos Estados Unidos, a partir da década de 1960. Atualmente engloba o conceito de Pharmaceutical Care, abrangendo todos os níveis de atenção à saúde (BRASIL, 2013a). No Brasil, seu início foi observado por meio do primeiro Serviço de Farmácia Clínica relatado em 15 de janeiro de 1979, no Hospital das Clínicas da Universidade Federal do Rio Grande do Norte, sendo aprimorada com o passar dos anos (BRANDÃO; CUNHA, 2010).

Pode ser definida como "área da farmácia voltada à ciência e prática do uso racional de medicamentos, na qual os farmacêuticos prestam cuidado ao paciente, de forma a otimizar a farmacoterapia, promover saúde e bem-estar, e prevenir doenças". Esta prática clínica pode ser desenvolvida em diferentes locais, como em hospitais, ambulatórios, unidades de atenção primária à saúde, farmácias comunitárias, domicílios entre outros (BRASIL, 2013a).

No Brasil a atuação clínica do farmacêutico vem sendo respaldada pela publicação de duas normativas que mudaram o cenário da atuação do profissional farmacêutico na assistência ao paciente. A Resolução do Conselho Federal de Farmácia n 585, de 29 de agosto de 2013 regulamenta as atribuições clínicas do farmacêutico. Por meio desta normatização ficam delimitados diferentes serviços clínicos que o farmacêutico pode prestar, como acompanhamento farmacoterapeutico, conciliação terapêutica, revisão da farmacoterapia entre outros, visando à promoção, proteção e recuperação da saúde (BRASIL, 2013a).

De maneira complementar as atribuições clínicas do farmacêutico foi aprovada por meio da resolução $n^{\circ} 585$, de 29 de agosto de 2013, a prescrição farmacêutica. Vale ressaltar que o farmacêutico poderá realizar "a prescrição de medicamentos e outros produtos com finalidade terapêutica, cuja dispensação não exija prescrição médica". Do contrário esta prescrição com restrição médica só poderá ocorrer quando formalizado por meio de protocolos ou normas técnicas internas ao local de trabalho (BRASIL, 2013b).

\subsection{A prática da Assistência e Atenção Farmacêutica}

No Brasil o conceito de Farmácia começa a mudar com a publicação da Lei n¹3.021, 
de 8 de agosto de 2014, que dispõe sobre o exercício e a fiscalização das atividades farmacêuticas e traz as disposições que norteiam ações e serviços da assistência farmacêutica (BRASIL, 2014).

A fim de garantir a assistência terapêutica integral, a assistência farmacêutica desenvolve um conjunto de ações e de serviços que visam como objetivo principal a promoção, proteção e recuperação da saúde no local em que o profissional farmacêutico busca o uso seguro e racional dos medicamentos (BRASIL, 2014). Por sua vez, a atenção farmacêutica consiste em um conjunto de práticas desenvolvidas pelo farmacêutico, que busca a orientação do paciente quanto ao uso correto de medicamentos (OPAS/OMS, 2002). Atendendo de maneira individualizada as necessidades dos pacientes (FAUS; MARTINEZ, 1999; SANTOS, et al., 2019).

Alguns artigos demonstram a importância da participação do farmacêutico clínico durante intervenções em prescrições médicas, evidenciando problemas relacionados a medicamentos (REIS, et al., 2013). Desta maneira, o farmacêutico atende, avalia e orienta o paciente em relação à farmacoterapia prescrita pelo médico (FURTADO, 2001), por meio da análise das suas necessidades relacionadas aos medicamentos, detectando também possíveis problemas relacionados a medicamentos (PERETTA; CICCIA, 2000). O que reforça a relevância de intervenções farmacêuticas em diferentes casos, buscando por mudanças positivas nas prescrições avaliadas, promovendo acima de tudo, promoção, proteção e recuperação a saúde (CIPOLLE, et al., 2000).

A consolidação do profissional farmacêutico na prática clínica vem se desenvolvendo com o passar dos anos por meio de estudos científicos e legislações que regulamentam a profissão. Nos quais ficam evidentes que o envolvimento e a participação dos farmacêuticos no atendimento aos pacientes trazem diversos benefícios (CHAMOUN,

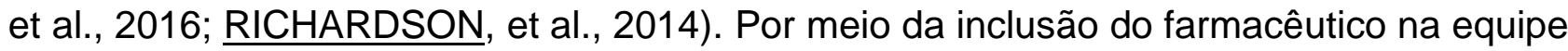
multiprofissional é possível identificar e abordar individualmente os problemas relacionados aos medicamentos (KJELDSEN, et al., 2014). Para que seja possível alcançar os objetivos propostos é preciso a participação e colaboração dos outros profissionais de saúde envolvidos (OKUMURA, et al., 2016).

\subsection{0 crescimento da atenção farmacêutica}

Ao pesquisar as publicações sobre o tema da prática dos cuidados farmacêuticos na base de dados Pubmed, foram encontrados 22.010 artigos inicialmente quando utilizado 
apenas o descritor "Pharmaceutical care practice". Alguns acontecimentos históricos auxiliaram no crescimento de estudos e pesquisas no segmento da prática clínica farmacêutica, acompanhando o aumento de publicações a nível mundial e nacional. Ao comparar as publicações utilizando os descritores "pharmaceutical care practice" em função do tempo, observa-se que os primeiros artigos foram publicados no ano de 1947 (3 artigos) (conforme GRÁFICO 1) os quais estão relacionados de maneira geral com a prescrição clínica.

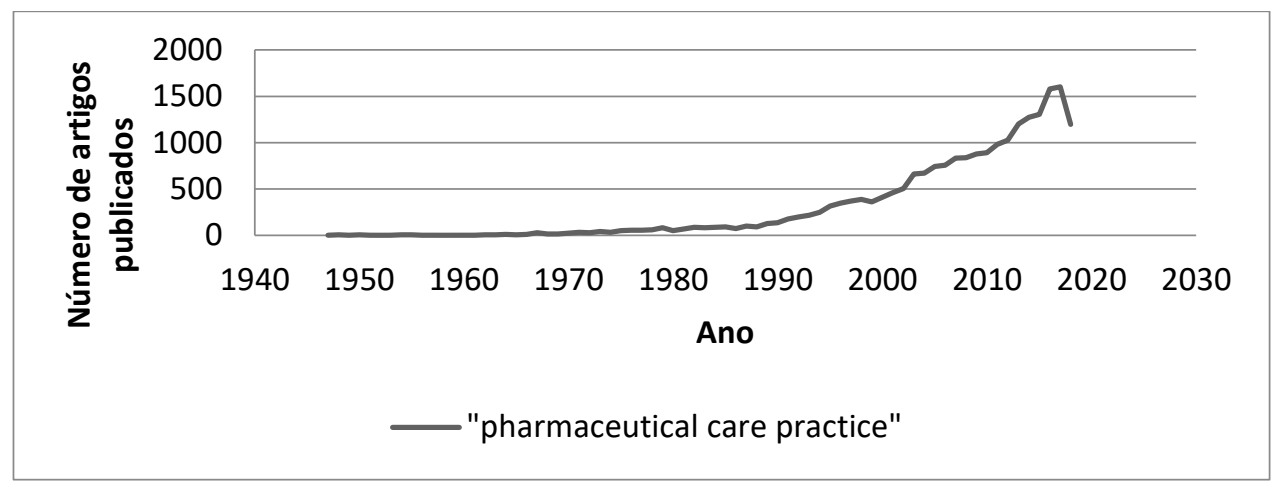

\section{GRÁFICO 1 - NÚMERO DE PUBLICAÇÕES A NÍVEL MUNDIAL EM FUNÇÃO DO ANO FONTE: A AUTORA (2019).}

Na década de 60 observou-se um discreto aumento no número de estudos. Um dos marcos deste período foi o início da farmácia clínica nos Estados Unidos, a partir da década de 1960, cujo principal objetivo voltava-se para a aproximação do farmacêutico ao paciente e à equipe de saúde.

Durante a década de 80 o número de publicações ainda era discreto, porém sempre em crescimento. Neste período o papel do farmacêutico em relação ao paciente passava por modificações, uma vez que a atuação do farmacêutico ainda não estava bem definida. Pode-se estabelecer um novo conceito para a época a "Atenção Farmacêutica", onde o farmacêutico participaria dos serviços necessários para proporcionar um tratamento farmacoterapêutico eficaz (BRODIE, et al., 1980).

Porém a partir da década de 90 pode-se observar um aumento mais expressivo, chegando a 387 artigos (1,76\% do total publicado) em 1998. Em 1990, a definição de Atenção farmacêutica passa a ser mais abrangente. Hepler e Strand citam em estudos científicos o termo "Pharmaceutical Care", que significa "Atenção Farmacêutica". Nesse artigo, os autores ressaltam a importância do farmacêutico na melhoria da qualidade de vida dos pacientes, ressaltando que "Atenção Farmacêutica é a provisão responsável do 
tratamento farmacológico com o objetivo de alcançar resultados satisfatórios na saúde, melhorando a qualidade de vida do paciente" (HEPLER; STRAND, 1990).

Visto a importância de tais acontecimentos a Organização Mundial de Saúde (OMS), discutiu, aceitou e ampliou a definição anterior, destacando que as funções na atenção farmacêutica são "o farmacêutico como dispensador da atenção sanitária que pode participar, ativamente, na prevenção das doenças e da promoção da saúde, junto com outros membros da equipe sanitária" (OMS, 1994).

Fica evidente o crescimento de publicações com o passar dos anos, sendo observados 1.579 artigos publicados no ano de 2016, o que corresponde a $7,17 \%$ do total de publicações no período avaliado. Os artigos referem-se a assuntos variados que incluem os problemas da automedicação, acompanhamento farmacoterapêutico, a expansão dos serviços de farmácia clínica, efeito de redução de custos e a prevenção de erros de medicação pela intervenção clínica do farmacêutico, dentre outros. Ao ser incluso o descritor "Brazil" à essa pesquisa ("Pharmaceutical care practice AND "Brazil") foram localizados 178 artigos, uma parcela ainda pequena desse total $(0,81 \%$ dos artigos obtidos a nível mundial) mas que vem crescendo com o decorrer dos anos, conforme observado no GRÁFICO 2.

Quanto ao cenário brasileiro (GRÁFICO 2), as publicações selecionadas com o descritor "pharmaceutical care practice AND Brazil" começaram a ser publicadas mais tardiamente quando comparamos com os resultados a nível mundial. O primeiro artigo encontrado no Brasil data de 1994 (1 artigo), ano em que se nota aumento nas publicações mundiais. Essa publicação relata padrões de intervenção médica em pacientes com AIDS na região sul do Brasil, não sendo o foco deste estudo.

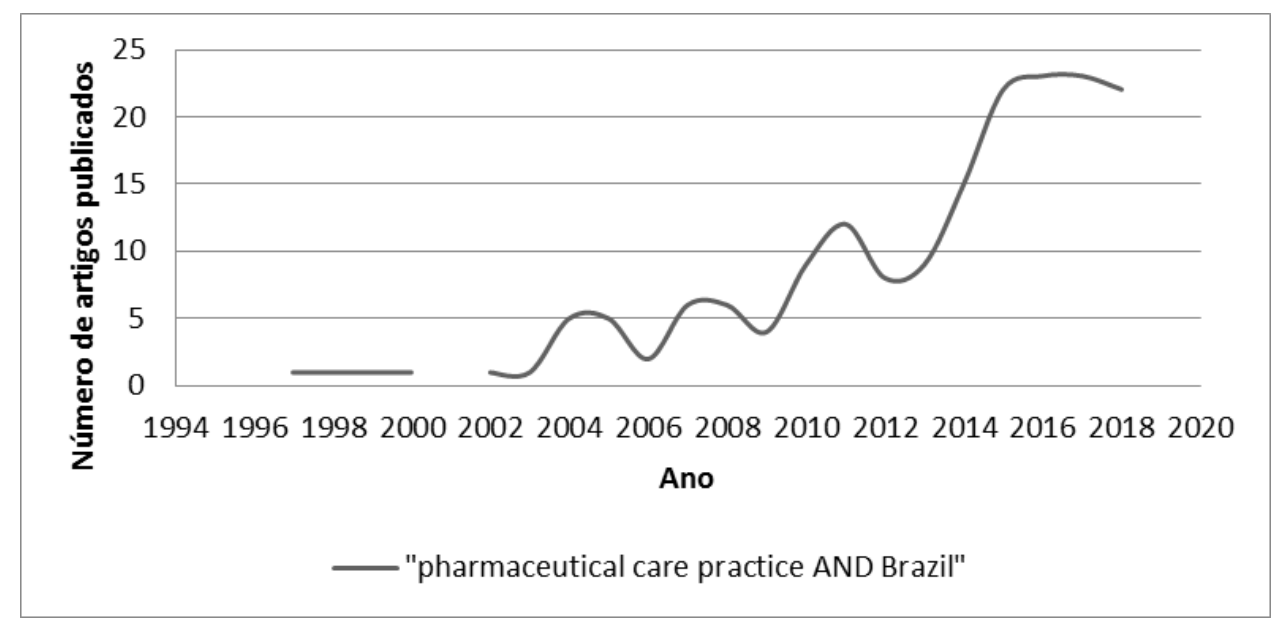

GRÁFICO 2 - NÚMERO DE PUBLICAÇÕES EM FUNÇÃO DO ANO NO BRASIL FONTE: A AUTORA (2019). 
Por volta de 2002 começam a surgir publicações mais específicas à área farmacêutica, como o artigo de Lieber e colaboradores (2002), que trata sobre uma revisão de literatura sobre intervenções farmacêuticas no uso de medicamentos por pacientes idosos, o qual demonstra que as intervenções realizadas pelo farmacêutico possuem resultados positivos, como redução de custos, maior adesão do paciente ao tratamento e controle a possibilidade de reações adversas.

Em 1994 a Organização Mundial de Saúde (OMS), revê a definição de atenção farmacêutica, o que contribui com avanços na área, por ampliar o papel do farmacêutico nos cuidados a saúde (OMS, 1994). Em 1998, a Portaria 3.916 estabelece a Política Nacional de Medicamentos, que reorienta a assistência farmacêutica, focando no uso racional de medicamentos (BRASIL, 1998).

A promoção da atenção farmacêutica no Brasil foi construída com base em discussões lideradas pela Organização Pan-Americana de Saúde/Organização Mundial da Saúde (OPAS/OMS, 2002). Nestes encontros foi definida a atenção farmacêutica como um modelo de prática farmacêutica, desenvolvida no contexto da Assistência Farmacêutica, cujo objetivo é prevenção de doenças, promoção e recuperação da saúde.

As publicações começam a se tronar mais expressivas em 2011, com 12 artigos. Que discutem algumas das atribuições clínicas do farmacêutico no cuidado com o paciente. O maior número de publicações pode ser observado em 2016, com 23 artigos (12,92\% dos artigos publicados), que abordam temas como o cuidado com incompatibilidade de medicamentos, a contribuição dos cuidados farmacêuticos no acompanhamento terapêutico e a eficácia da participação do farmacêutico na adesão ao tratamento.

Ao longo dos anos percebe-se que os avanços obtidos na área da farmácia clínica em nosso país está intimamente relacionada com as conquistas obtidas, pois a partir de 2013 o Conselho Federal de Farmácia regulamenta as atribuições clínicas do farmacêutico delimitando diferentes serviços clínicos que o farmacêutico pode prestar, como acompanhamento farmacoterapeutico, conciliação terapêutica, revisão da farmacoterapia entre outros. Desta maneira, a farmácia clínica tem a Atenção Farmacêutica como uma ferramenta de suporte, sendo uma complementar a outra, que facilita a interação do farmacêutico com o paciente (BRASIL, 2013c).

\section{CONCLUSÃO}

Diversos estudos apontam a expansão e interesse no desenvolvimento de pesquisas 
cientificas que envolvam a prática clínica, atenção e cuidados farmacêuticos no Brasil e no mundo. É evidente a participação do profissional farmacêutico no cuidado a saúde dos pacientes tanto mundialmente quanto a nível nacional.

A intervenção e os demais serviços clínicos prestados pelo farmacêutico que compõem a equipe multidisciplinar de estabelecimentos de saúde reforçam o crescimento e evolução da profissão de maneira significativa. Quando observamos o número de artigos publicados que abordam as práticas da atenção farmacêutica fica claro o expressivo crescimento com o passar dos anos. Desta maneira, o estudo das atribuições do farmacêutico de maneira geral mostra-se um campo promissor em pesquisas cientificas.

\section{REFERÊNCIAS}

ANGONESI, D.; SEVALHO, G. Atenção farmacêutica: fundamentação conceitual e crítica para um modelo brasileiro. Ciências e Saúde Coletiva. n. 15, supl. 3, p. 3613-3614, 2010.

BRANDÃO, A.; CUNHA, C. Farmácia clínica: sonho, realização e história. Pharmacia Brasileira. Maio/Junho 2010.

BRASIL. Ministério da Saúde. Portaria 3.916, de 30 de outubro de 1998. Aprova a Política Nacional de Medicamentos. Diário Oficial da União, Poder Executivo, Brasília, DF, 10 dez. 1998. Seção 1, p. 18.

BRASIL. CONSELHO FEDERAL DE FARMÁCIA. Resolução nำ585 de 29 de agosto de 2013. Ementa: Regulamenta as atribuições clínicas do farmacêutico e dá outras providências. Diário Oficial da União, Poder Executivo, Brasília, DF, 29 ago. 2013a.

BRASIL. CONSELHO FEDERAL DE FARMÁCIA. Resolução nำ586 de 29 de agosto de 2013. Ementa: Regula a prescrição farmacêutica e dá outras providências. Diário Oficial da União, Poder Executivo, Brasília, DF, 29 ago. 2013b.

BRASIL. CONSELHO FEDERAL DE FARMÁCIA. Resolução nำ 585 de 29 de agosto de 2013. Regulamenta as atribuições clínicas do farmacêutico e dá outras providências. Diário Oficial da União, Poder Executivo, Brasília, DF, 29 ago. 2013c. 
BRASIL. PRESIDÊNCIA DA REPÚBLICA. Lei n 13.021 de 8 de agosto de 2014. Dispõe sobre o exercício e a fiscalização das atividades farmacêuticas. Diário Oficial da União, Poder Executivo, Brasília, DF, 8 ago. 2014.

BRODIE, D. C.; PARISH, P. A.; POSTON, J. W. Societal needs for drugs and drug-related services. Am. J. Pharm. Educ., v.44, n.3, p.276-78, 1980.

CARREÑO, M. G.; CRESPO, I. R.; et al. Optimización de la farmacoterapia en un hospital de Traumatología. Farm Hosp. v.36, n.1, p.16-23.2012

CHAMOUN, N. R.; ZEENNY, R.; MANSOUR, H. Impact of clinical pharmacy interventions on medication error nodes. Int J Clin Pharm. v.38, n.6, p.1436-1444. 2016.

CIPOLLE, D.J., STRAND, L. M., MORLEY, P.C. El ejercicio de la atención farmacéutica Madrid: McGraw Hill / Interamericana, p. 1-36, 2000.

COSTA, K. S.; et al. Coordinación entre servicios farmacéuticos para una farmacoterapia integrada: el caso de Cataluña. Ciênc. Saúde Coletiva, v.22, n.8, P. 2595-2608. 2017.

FAUS, M.J., MARTINEZ, F. La atención farmacéutica en farmacia comunitaria: evolución de concepos, necesidades de formación, modalidades y estratégias para su puesta en marcha. Pharm. Care Esp. v. 1, p. 56-61, 1999.

FONSECA, J. J. S. Metodologia da pesquisa científica. Fortaleza: UEC, 2002.

FURTADO, G. R. Noções Básicas sobre Atenção Farmacêutica. Curitiba: UFPR, 2001.

GERHARDT, T. E.; SILVEIRA. D. T. Métodos de pesquisa. Porto Alegre: Editora da UFRGS, 2009.

HEPLER, C.D.; STRAND, L.M. Opportunities and responsibilities in pharmaceutical care. Am. J. Hosp. Pharm., v.47, n.3, p.533-543, 1990.

KJELDSEN, L. J.; CLEMMENSEN, M. H.; et al. Evaluation of a controlled, national collaboration study on a clinical pharmacy service of screening for risk medications. Int $\mathbf{J}$ Clin Pharm. v.36, n.2, p.368-76. 2014. 
LIEBER, N. S. R.; TEIXEIRA, J. J. V.; FARHAT, F. C. L. G. et al. Revisão dos estudos de intervenção do farmacêutico no uso de medicamentos por pacientes idosos. Cad. Saúde Pública, v.18, n.6, p. 1499- 1507. 2002

OKUMURA, L. M.; SILVA, D. M.; COMARELLA, L. Relação entre o uso seguro de medicamentos e Serviços de Farmácia Clínica em Unidades de Cuidados Intensivos Pediátricos. Rev. Paul. Pediatr. v.34, n.4. P. 397-402. 2016.

ORGANIZAÇÃO MUNDIAL DE SAÚDE (OMS). The role of the pharmacist in the health care system. Geneva: OMS, 1994. 24p. (Report of a WHO Meeting).

ORGANIZAÇÃO PANAMERICANA DA SAÚDE; ORGANIZAÇÃO MUNDIAL DA SAÚDE (OPAS/OMS). Relatório 2001-2002: atenção farmacêutica no Brasil: trilhando caminhos. Brasília, 2002.

PERETTA, M.; CICCIA, G. Reengenharia farmacêutica - guia para implantar atenção farmacêutica. Brasília: Ethosfarma, 2000.

REIS, W. C.; SCOPEL, C. T.; CORRER, C. J.; ANDRZEJEVSKI, V. M. Análise das intervenções de farmacêuticos clínicos em um hospital de ensino terciário do Brasil. Einstein., v.11, n.2, p. 190-6. 2013.

RICHARDSON, T. E.; O'REILLY, C. L.; CHEN, T. F. A comprehensive review of the impact of clinical pharmacy services on patient outcomes in mental health. Int J Clin Pharm. n.36, v.2, p.222-32. 2014.

SANTOS, N. S.; MARENGO, L. L.; MORAES, F. S.; BARBERATO, S. Intervenções para reduzir a prescrição de medicamentos inapropriados para idosos. Rev. Saúde Pública., n.7, v.53, p.1-16. 2019. 